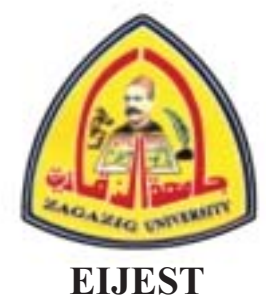

The Egyptian Int. J. of Eng. Sci. and Technology

Vol. 17, No. 2 (April 2014)

\title{
A NUMERICAL INCREMENTAL PROCEDURE FOR SOLVING NON-LINEAR ELASTOPLASTIC FRICTIONAL CONTACT PROBLEMS UNDER NORMAL LOAD*
}

\author{
Waleed S. Abdalla ${ }^{+}$, Soliman S. Ali-Eldin, Mohamed R. Ghazy \\ Department of Mechanical Design and Production Engineering, \\ Faculty of Engineering, Zagazig University, Zagazig 44511, EGYPT
}

\begin{abstract}
This paper presents an adaptive incremental approach capable of handling elastoplastic contact problems with friction. Using the finite element method (FEM), the problem is formulated as an incremental convex programming model (ICPM) under the inequality contact constraints and friction conditions. The Lagrange multiplier approach is adopted for imposing the contact constraints. The classical Coulomb's law of friction is used for simulating the friction conditions throughout the contact interface. The incremental constitutive relations based on the Prandtl-Reuss equations and von Mises yield criterion are adopted to simulate the elastoplastic response. The adopted model advantage is that the model gets rid of the inclusion of any artificial element or intervene user defined parameters such as penalty values and the number of load steps. Numerical examples for the frictional contact having advancing and receding nature under normal load are presented. Illustrated examples prove the capability of the incremental procedure to investigate the sequence of different events during monotonic application of external load. Variations of the contact pressure, relative tangential displacement (RTD), tangential stress over the contact area and equivalent stress within the domain with the coefficient of friction are presented to validate the model. Good agreement has been found with published results.
\end{abstract}

KEY WORDS: Nonlinear, Elastoplastic Contact, Friction Coulomb Law, Convex Programming, FEM.

\section{RÉSUMÉ}

Cet article présente une approche progressive adaptative capable de gérer les problèmes de contact avec frottement élasto-plastiques. Utilisation de la méthode des éléments finis (FEM), le problème est formulé comme un modèle de programmation convexe incrémentale (CIMP) sous les contraintes de contact de l'inégalité et conditions de frottement. L'approche des multiplicateurs de Lagrange est adoptée pour imposer les contraintes de contact. La loi de Coulomb classique de frottement est utilisé pour simuler les conditions de frottement dans l'interface de contact. Les relations constitutives supplémentaires basés sur les équations de Prandtl-Reuss et von Mises critère de rendement sont adoptées pour simuler la réponse élasto-plastique. L'avantage du modèle adopté est que le modèle se débarrasse de l'inclusion de tout élément artificiel ou intervenir les paramètres définis par l'utilisateur tels que les valeurs de pénalité et le nombre de prises en charges. Des exemples numériques pour le contact de friction ayant entrants et sortants nature sous une charge normale sont présentés. Exemples illustrés prouvent la capacité de la procédure incrémentale à rechercher la séquence d'événements différents lors de l'application monotone de charge externe. Les variations de la pression de contact, le déplacement tangentiel relatif (RTD), contrainte tangentielle sur la surface de contact et la contrainte équivalente au sein du domaine avec le coefficient de frottement sont présentés pour valider le modèle. Un bon accord a été trouvé avec les résultats publiés.

MOTS CLÉS: Non Linéaires, Élasto-Plastique De Contact, Friction Loi De Coulomb, Programmation Convexe, FÉM.

* Received: 27/1/2014, accepted: 17/2/2014, Ref. No. 178, (original paper)

+ Contact author (+201222359080, waleed_zaraa@yahoo.com) 


\section{INTRODUCTION}

Contact mechanics is a fundamental discipline of the engineering sciences, which is indispensable for the construction of safe designs. Contact problems between deformable bodies involve complicated surface phenomena, are modeled by strongly nonlinear boundary value problems. Introduction of the material nonlinearity and friction are additional formidable complications as they represent an irreversible processes that make the contact problems of deformable bodies highly nonlinear and much more difficult.

In the literature, many numerical approaches had developed to deal with contact problems that were based on the incremental and iterative methods. Using the load incremental theory, Okamoto and Nakazaw [1] developed an incremental method for the solution of elastic frictional contact problems using classical Coulomb friction law. Oden and Pires [2] proposed nonlocal and nonlinear friction law. The nonlocality was attributed to the pattern of pressure distribution over deformed asperities. The nonlinearity resulted from the occurrence of relative micro-displacements between contact pair nodes before the gross sliding of the two contacting surfaces. Bathe and chaudhary [3] formulated an incremental method with iterative technique for the analysis of planar and axisymmetric contact problems using FEM and Coulomb's law of friction. The contact conditions were imposed by considering the total potential of contact forces with the geometric compatibility conditions. Zhong and Sun [4] developed a numerical procedure for solving an elastic contact problem with Coulomb's friction using the parametric quadratic programming. The formulation was based on the FEM and penalty method for the introduction of contact constraints. Paris and Garrido [5], using the boundary element method (BEM), proposed an incremental procedure to solve the frictional contact problems between elastic deformable bodies using Coulomb's friction law.

Garrido et al. [6] extended Paris and Garrido [5] through an integral boundary formulation of the three-dimensional friction contact problem. Lee [7] developed a numerical procedure for the solution of 2-D frictional contact problems using FEM with Coulomb's friction law. Saleeb et al. [8] developed a FE model for analysis of two dimensions static frictional contact problems, based on perturbed Lagrange type and the classical Coulomb frictional law. Mahmoud et al. [9] proposed an adaptive incremental approach to solve multiphase elastic frictional contact problems using the non-classical local nonlinear friction law.

Mijar and Arora [10] formulated the frictional contact problems as variation equality using an augmented Lagrangian method (ALM). Renaud and Feng [11] presented a comparative study of boundary element method (BEM) and FEM for the analysis of two dimensional elastostatic Signorini contact problems using Coulomb's friction law and penalty method. Hassan and Mahmoud [12] developed an incremental model for the solution of a general class of convex programming problems. These problems have linear constraints with zero and nonzero free coefficients. The incremental procedure was applied to solve the elasto-static frictionless contact problems. Mohamed et al. [13] proposed an elastic incremental FE model to simulate the frictional contact using non-classical friction model. The friction effect was represented by an equivalent nonlinear stiffness rather than additional constraints. Qin and Wang [14] suggested an incremental iterative scheme to solve frictional contact problems using a hybrid Trefftz FE formulation and the Coulomb friction Law. Ahn and Barber [15] formulated the receding contact problem with the classical Coulomb's friction law for an elastic block against a rigid surface using the FEM. They concluded that all the concepts of receding contact stated by Dundur's [16] can be extended to frictional receding contact problem as long as the loading is monotonic. Kanber and Demirhan [17] analyzed the problem of elastic thin-coated contact surfaces under friction or frictionless conditions. They used the theory of thin coating contact mechanics and ANSYS F. E commercial package. All models analyzed using the NewtonRaphson solution algorithm under static loading conditions. Abdalla et al. [18] developed an adaptive incremental procedure to solve the elastic friction advancing or receding contact problems based on the classical Coulomb's friction law and FEM. The problem was formulated as a convex programming model (CPM) under the inequality contact constraints and friction conditions using the Lagrange multiplier approach. 
The incremental method for solving elastoplastic problems by FEM based on PrandtlReuss equations and von Mises yield criterion was developed. Yamada et al. [19] presented an incremental solution algorithm based on the piecewise linearization of the equations for each load increment. The piecewise linearization technique used results at the end of the previous increment without any iteration. Zinckiewiz et al. [20] proposed a general formulation of the elastoplastic matrix relates the increments of stresses and strains for any yield surface with associated flow rule and for work hardening and ideal plastic material through an iterative solution technique.

Dumas and Baranet [21] solved the plane strain frictionless indentation of rigid cylinder and elastoplastic half-space using FEM. They concluded that with increasing load, the pressure distribution changed from elliptical to a rather flat shape. Nagaraj [22] studied the interaction of real surfaces by the simulation of asperities contact. The asperity contact was modeled as a rigid cylinder contacting an elastoplastic half-space with high friction coefficient to prevent the gross slip. Cheng and Kikuchi [23] proposed an incremental procedure, based on of the updated Lagrangian formulation, to solve the elastoplastic large deformation contact problem using PrandtlReuss equations and Coulomb's friction law. Komvopoulos [24] studied the frictional elasticplastic indentation contact problem of a layered half-space and a rigid cylinder using the 3-node, interface gap elements. Wang et al. [25] solved an elastoplastic contact problem of Vickers indentation using the FEM through an incremental-iterative technique. Montmitonnet et al. [26] studied the elastoplastic indentation, with or without friction, of half-space by spherical indenter using isoparametric FE analysis. They found that for frictionless elastoplastic contact, the contact pressure profile changed from elliptic to nearly flat and did not greatly affected by friction. Zhu [27] presented finite element mathematical programming method to solve elastoplastic contact problems with friction. The problem formulated based on virtual work, which was applied to elastic-perfectly plastic problems.

Pantuso et al. [28] presented a solution procedure for the analysis of thermo-mechanical conditions of solids in contact with large plastic deformations adopting Coulomb friction model. The contact constraints imposed using Lagrange multiplier method. Menezes and Teodosiu [29] presented a 3-D model for the numerical simulation of the frictional contact problem of deep-drawing process, using the FEM and Coulomb's classical law through an augmented Lagrangian formulation. The large elastoplastic strains and rotations with anisotropic plastic properties of the sheet was considered in the model. Sheng et al. [30] presented numerical formulation for frictional contact problems associated with pile penetration using the theory of hardening/softening plasticity. The formulation and numerical procedure based on the FEM and the classical Coulomb frictional law with automated load stepping scheme. Vijaywargiya and Green [31] used ANSYS FE commercial Package to simulate 2D sliding between two interfering elastic-perfectly plastic cylinders that follows the von Mises yield criterion. The results provided the deformations, reaction forces, and the stresses. Anahid and Khoei [32] modeled arbitrary interfaces with large elastoplastic deformation. The model was based on the X-FEM coupled with the Lagrangian formulation and the incremental elastoplastic stress-strain relation. They applied the model to the crack propagation problem without remeshing.

Bandeira et al. [33], using the FEM with exact linearization, formulated the 3D contact problems with friction undergo large deformation within an elastoplastic range using ALM and a node-tosurface algorithmic. For sliding region, the nodeto-surface contact formulation was not sufficient, and special cases of node-to-edge and node-tonode contact formulations were employed. Adam and Swain [34] used ABAQUS to explore the effect of friction in simulation of bone nanoindentation using spheroconical indenter. An axisymmetric FE simulation of the elasticperfectly plastic frictional contact problem was performed with Dracker-Parger and von-Mises yield surfaces. Boudaia et al. [35] presented a theoretical and FE analysis of elastoplastic frictional contact problems for large deformation. Coulomb's friction law and Penalty method used to simulate the contact constraints. Chatterjee and Sahoo [36] studied the effect of strain hardening on elastic-plastic contact of a sphere with a rigid flat under full stick contact condition. They used ANSYS with different bilinear isotropic hardening. They found that under a full stick contact condition, strain hardening greatly influenced the contact parameters, i.e., pressure and area, and the contact conditions had negligible effect in comparison between perfect slip and stick contact. Weyler et al. [37] 
formulated the friction contact problem with large deformation and plastic behavior. The Lagrange multiplier and the penalty strategies were used to impose the contact constraints. They concluded that the use of the Lagrange multiplier based strategy had an advantage in a few critical situations, where the penalty method failed to produce convincing results due to excessive penetration. Kumar et al. [38] studied experimentally the influence of applied normal load and the roughness on the tribological behavior during nanoindentation contact. They observed that the friction coefficient was constant during elastic contact regime and increased with increasing load when the contact involved plastic deformation. The transition load from elastic to plastic contact increased with increasing the size of the indenter and decreased with roughness. Poulios and Klit [39] presented a FE model for frictionless conformal contact of nominally flat rough surfaces using Lagrange multiplier method, and elastic and perfectly elastic-plastic materials. Deformation, contact pressure and real contact area were obtained under normal load only.

The objective of this study is to investigate the frictional contact of elastoplastic solids with strain hardening due to static normal load using the classical Coulomb's friction law. Based on the FEM, the contact problems presented as an incremental convex programming model that is solved using an adaptive incremental procedure where contact and friction constraints are handled using the Lagrange multiplier method. The solved examples, the contact of a cylinder that is pressed against a rigid support and the contact of a block with deformable foundation, are illustrated. Distribution of the contact pressure, tangential stress, RTD over the contact area, and the distribution of equivalent stress inside the domain are presented while varying the coefficient of friction. The obtained results are compared with the available results in the literature, and good agreements are obtained.

\section{GOVERNING RELATIONSHIPS}

In this section, we shall discuss the relationships that describe the physical phenomena of friction by Coulomb law and the elastoplastic behavior of the material through the Prandtl-Reuss flow rule.

\subsection{Coulomb's Friction Law}

One of the most popular friction laws is Coulomb's friction law, which states that the friction force is proportional to the normal applied force, Popov [40];

$F_{t} \leq \mu F_{n}$

where $F_{t}$ and $F_{n}$ are the tangential (frictional) force and the normal force, respectively, and $\mu$ defines the static coefficient of friction. The contact zone can be divided into two parts; sticking and slipping. Therefore, the contact status can be specified according to the tangential force as follows:

$\left|F_{t}\right| \begin{cases}<\mu F_{n} & \text { sticking and } u_{t}=0 \\ =\mu F_{n} & \text { slip and } \exists \eta \geq 0, u_{t}=-\eta \sigma_{t} \neq 0\end{cases}$

where $u_{t}$ is the relative tangential displacement and $\sigma_{t}$ is the tangential stress, which is related to the normal stress, $\sigma_{n}$, by

$\sigma_{t}=-\mu \sigma_{n} u_{t} /\left|u_{t}\right|$

In Eq. (3), the negative sign means that the tangential friction force acts in a direction opposite to the relative motion.

\subsection{Elastoplastic Incremental Stress-Strain Relations}

The incremental plastic strain-stress relationship in its general form is given by, Mendelson [41];

$d \varepsilon_{i j}^{p}=d \lambda \frac{\partial f}{\partial \sigma_{i j}}$

where $d \varepsilon_{i j}^{p}$ is the plastic strain increment tensor, $\sigma_{i j}$ is the stress tensor; $f$ is the loading function that depends upon the chosen yielding criterion, and $d \lambda$ is a nonnegative constant that depends upon the loading history and the strain hardening properties of the material.

For the von Mises yielding criterion, the relation between the increments of plastic strain and stress that known as Prandtl-Reuss flow rule is given by 
$d \varepsilon_{i j}^{p}=\frac{3}{2} \frac{d \sigma_{e}}{H^{\prime} \sigma_{e}} S_{i j}$

in which

$$
\sigma_{e}=\sqrt{\frac{3}{2} S_{i j} S_{i j}}, d \varepsilon_{p}=\sqrt{\frac{2}{3} d \varepsilon_{i j}^{p} d \varepsilon_{i j}^{p}} \text { and } H^{\prime}=\frac{d \sigma_{e}}{d \varepsilon_{p}}
$$

where $S_{i j}$ is the current stress deviator tensor, $S_{i j}=\sigma_{i j}-\delta_{i j} \sigma_{k k} / 3 . \sigma_{e}$ is the current equivalent stress, $d \varepsilon_{p}$ is the equivalent plastic strain increment, and $H^{\prime}$ is the slope of uniaxial stressplastic strain curve. The increment of equivalent stress, $d \sigma_{e}$, can be expressed as

$d \sigma_{e}=\frac{\partial \sigma_{e}}{\partial \sigma_{i j}} d \sigma_{i j}$

Assuming small displacements and rotations, the total strain increment can be additively decomposed;

$d \varepsilon_{i j}=d \varepsilon_{i j}^{e}+d \varepsilon_{i j}^{p}$

where $d \varepsilon_{i j}{ }^{e}$ and $d \varepsilon_{i j}{ }^{p}$ are the components of elastic and plastic strain increments, respectively. The elastic incremental strain-stress relationship is simulated using Hooke's law;

$d \varepsilon_{i j}^{e}=C_{i j k m}^{e} d \sigma_{k m}$

where $C_{i j k m}^{e}$ is the elasticity compliance matrix, depends only on the modulus of elasticity, $E$, and Possion ratio, $v$, for isotropic materials, which can be inverted to give stress-strain elasticity matrix, $D_{i j k m}^{e}$.

Assuming small deformations, the engineering incremental strian-displacement relations are given by

$\Delta \varepsilon_{i j}= \begin{cases}0.5\left(\Delta u_{i, j}+\Delta u_{j, i}\right) & \text { for } i=j \\ \left(\Delta u_{i, j}+\Delta u_{j, i}\right) & \text { for } i \neq j\end{cases}$

where $\Delta u_{i}$ are the displacement increment components.

Using Eqs. (5-10), the explicit matrix form of the elastoplastic incremental strain-stress relations are derived in appendix (A) that represented by Eqs. (A.2), (A.3) and (A.6) for different cases. The increments of total strain and increments of stress are related through the elastoplastic compliance matrix. It is evident that the source of the nonlinearty is due to the dependence of the elastoplastic compliance matrix coefficients on values of the current (total) stresses, which depend on the values of the unknown stress increments.

The elastoplastic incremental stress-strain relation is given by

$\Delta \sigma_{i j}=D_{i j k m}^{e p} \Delta \varepsilon_{k m}$

where $D_{i j k m}^{e p}$ is the elastoplastic matrix that depends upon the loading history and the strain hardening properties of the material.

\subsection{Yield Scale Factor}

In the incremental method, which will be described later, it is need to scale the stresses within an element that will change from the elastic to the plastic state in order to induce it to the onset of yield.

Let $\sigma_{x}, \sigma_{y}, \sigma_{z}$ and $\tau_{x y}$ be the stresses at the beginning of the increment, and $\Delta \sigma_{x}, \Delta \sigma_{y}, \Delta \sigma_{z}$ and $\Delta \tau_{x y}$ be the stress increments due to the application of the load.

For plane stress analysis let,

$$
\begin{aligned}
& \bar{\sigma}_{e}= \sigma_{x}^{2}+\sigma_{y}^{2}+3 \tau_{x y}^{2}-\sigma_{x} \sigma_{y} \\
& D=2 {\left[\sigma_{x} \Delta \sigma_{x}+\sigma_{y} \Delta \sigma_{y}+3 \tau_{x y} \Delta \tau_{x y}\right.} \\
&\left.-0.5\left(\sigma_{x} \Delta \sigma_{y}+\sigma_{y} \Delta \sigma_{x}\right)\right] \\
& \Delta \sigma_{e}=\Delta \sigma_{x}^{2}+\Delta \sigma_{y}^{2}+3 \Delta \tau_{x y}^{2}-\Delta \sigma_{x} \Delta \sigma_{y}
\end{aligned}
$$

Also, for plane strain case, let

$$
\begin{gathered}
\bar{\sigma}_{e}=\sigma_{x}^{2}+\sigma_{y}^{2}+\sigma_{z}^{2}-\sigma_{x} \sigma_{y}-\sigma_{y} \sigma_{z}-\sigma_{x} \sigma_{z} \\
+3 \tau_{x y}^{2} \\
\mathrm{D}=2 \sigma_{x} \Delta \sigma_{x}+2 \sigma_{y} \Delta \sigma_{y}+2 \sigma_{z} \Delta \sigma_{z}-\left(\sigma_{z}+\sigma_{y}\right) \Delta \sigma_{x} \\
-\left(\sigma_{x}+\sigma_{z}\right) \Delta \sigma_{y}-\left(\sigma_{x}+\sigma_{y}\right) \Delta \sigma_{z}+6 \tau_{x y} \Delta \tau_{x y} \\
\Delta \sigma_{e}=\Delta \sigma_{x}^{2}+\Delta \sigma_{y}^{2}+\Delta \sigma_{z}^{2}-\Delta \sigma_{x} \Delta \sigma_{y}-\Delta \sigma_{y} \Delta \sigma_{z} \\
-\Delta \sigma_{z} \Delta \sigma_{x}+3 \Delta \tau_{x y}^{2}
\end{gathered}
$$

Therefore, the current value of the equivalent stress within an elastic element tends to be plastic is given by 
$\sigma_{e}^{2}=\bar{\sigma}_{e}+\Delta \sigma_{e}+D$

At the onset of yielding, the equivalent stress, $\sigma_{e}$, have to be equal to the yield stress, $S_{y}$. Thus

$S_{y}^{2}=\bar{\sigma}_{e}+S_{p} D+S_{p}^{2} \Delta \sigma_{e}$

where $S_{p}$ is the yield or the plasticity scale factor that for plane stress or plane strain is given by

$S_{p}=\frac{-D+\sqrt{D^{2}+4 \Delta \sigma_{e}\left(S_{y}^{2}-\sigma_{e}^{2}+\Delta \sigma_{e}+D\right)}}{2 \Delta \sigma_{e}}$

\section{MATHAMTICAL FORMULATION OF ELASTOPLASTIC FRICTIONAL CONTACT PROBLEM}

Consider two deformable bodies having an elastoplastic strain hardening material that are subjected to an external load and coming into contact with each other, as shown Figure (1). Each of the two bodies occupies a bounded domain $\bar{\Omega}$ in $R^{N}, N=1,2,3$ with open interior $\Omega$. The boundary $\Gamma$ of each body is assumed to consist of three disjoint parts: $\Gamma_{\mathrm{U}}, \Gamma_{\mathrm{t}}$, and $\Gamma_{\mathrm{C}} . \Gamma_{\mathrm{U}}$ is a part of the boundary at which displacements are prescribed. $\Gamma_{t}$ is a part of the boundary at which boundary tractions (forces) are prescribed. $\Gamma_{\mathrm{C}}$ is a part of the boundary that contains the contact constraints.

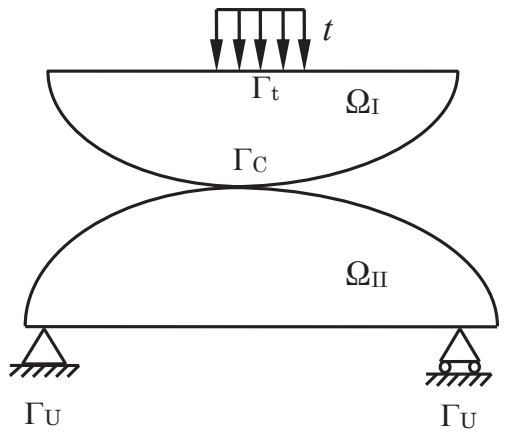

Fig. (1) A schematic of two bodies in contact.

The classical formulation of the frictional contact problem is presented as follows:

The static equilibrium equations of particles within $\Omega$ are given by

$\Delta \sigma_{i j, j}+\Delta f_{i}=0 \quad$ in $\Omega$ where $\Delta \sigma_{i j}$ is the stress increment tensor and $\Delta f_{i}$ are the body force increments per unit volume.

The incremental material constitutive model is given by

$\Delta \sigma_{i j}=D_{i j k m} \Delta \varepsilon_{k m} \quad$ in $\Omega$

where $\Delta \varepsilon_{\mathrm{km}}$ is the engineering strain increment tensor and $D_{i j k m}$ is given by $D_{i j k m}^{e}$ or $D_{i j k m}^{e p}$ for elastic region or plastic region, respectively. By assuming small deformations, the engineering incremental strian-displacement relationship is given by Eq. (10).

The boundary conditions are as follows:

$\begin{array}{ll}\Delta u_{i}=\bar{U} & \text { on } \Gamma_{\mathrm{U}} \\ \Delta t_{i}=\Delta \sigma_{i j} n_{j} & \text { on } \Gamma_{\mathrm{t}}\end{array}$

where $n_{j}$ are the components of the unit outward normal to $\Gamma$ and $\Delta t_{i}$ are the surface tractions.

The contact constraints on the contact interface are:

1. The normal contact constraints: the relative normal displacement between the candidate contact pair nodes on the surfaces of the two bodies I and II are given by

$\Delta U_{n}=\left(\Delta u_{i} n_{i}\right)^{I I}-\left(\Delta u_{i} n_{i}\right)^{I}=C_{n}^{T} \Delta u \quad$ on $\Gamma_{\mathrm{C}}$

The unilateral contact law takes the following form:

$$
\begin{array}{ll}
\Delta U_{n} \leq b \text { or } T_{n}=C_{n}^{T} \Delta u-b \leq 0 & \text { on } \Gamma_{\mathrm{C}} \\
\Delta \sigma_{n}=\Delta \sigma_{i j} n_{i} n_{j} \leq 0 & \text { on } \Gamma_{\mathrm{C}} \\
\Delta \sigma_{n}\left(C_{n}^{T} \Delta u-b\right)=0 & \text { on } \Gamma_{\mathrm{C}}
\end{array}
$$

The free coefficient vector, $b$, representing the gap between the contact nodes is greater than or equal to zero for advancing and receding contacts, respectively. $T_{n}$ is a vector that indicates the violation of the normal contact. In other words, a positive nonzero value of any of its components points out to a violation of the contact constraint. The violation means that the relative normal displacement exceeds the initial gap, which is prohibited as be noticed in Eq. (22).This equation demands that contactor and 
target cannot penetrate each other, i.e., impenetrability condition. Eq. (23) shows that the normal stress vector and hence the contact force at the contact region is compressive. The complementary condition repesented by Eq. (24) indicates that in the case of separation, we have $\Delta \sigma_{n}=0,\left(C_{n}^{T} \Delta u-b\right)<0$ and in the case of contact, we have $\Delta \sigma_{n} \leq 0,\left(C_{n}^{T} \Delta u-b\right)=0$. The unilateral contact law can be repesented graphically as shown in Figure (2).

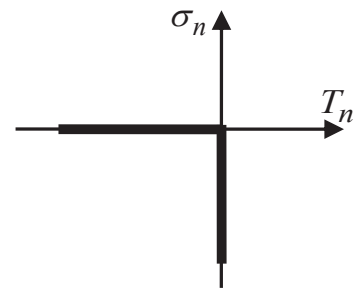

Fig. (2) A representation of the unilateral contact law.

2. The tangential contact constraints: the relative tangential displacement increment between pair nodes on the surfaces of the two bodies I and II, having tangetnial displacement increments $\Delta u_{t}^{I}$ and $\Delta u_{t}^{I I}$, respectively, is given by

$$
\begin{array}{ll}
\Delta U_{t}=\Delta u_{t}^{I}-\Delta u_{t}^{I I}=C_{t}^{T} \Delta u & \text { on } \Gamma_{\mathrm{C}} \\
T_{t}=\xi\left(T_{n}\right)\left|C_{t}^{T} \Delta u\right| \geq 0 & \text { on } \Gamma_{\mathrm{C}}
\end{array}
$$

where $T_{t}$ is a vector that indicates the violation in the tangential direction. The coefficient matrices shown in Eqs. (21), (22) and (25) for normal and tangential contact constraints are given by

$$
\begin{aligned}
& C_{n}=\left[\begin{array}{lllll}
C_{1-n} & C_{2-n} & C_{3-n} & \ldots & C_{N c-n}
\end{array}\right] \\
& C_{t}=\left[\begin{array}{lllll}
C_{1-t} & C_{2-t} & C_{3-t} & \ldots & C_{N c-t}
\end{array}\right]
\end{aligned}
$$

where $N_{C-n}$ is the number of candidate contact constraints, $N_{C-t}$ is the number of tangential contact constraints, and $C_{i-n}$ and $C_{i-t}$ are column vectors. The design variable $u$ is defined $U \in \delta$, where $\delta$ is a set defined as

$$
\delta=\left\{U: U \in H^{1}(\Omega)^{N}, \mathrm{U}=\overline{\mathrm{U}} \text { on } \Gamma \mathrm{U}\right\}
$$

where $H^{l}$ is the Hielbert space continous to the first order and $N=1,2$.

The unit step function $\xi\left(T_{n}\right)$ is defined by

$\xi\left(T_{n}\right)=\left\{\begin{array}{lll}1 & \text { if } & T_{n} \geq 0 \\ 0 & \text { if } & T_{n}<0\end{array}\right.$

The inequality constraints given by Eq. (26) can be interpreted as follows: when $\xi\left(T_{n}\right)=1$, there are two cases. If $T_{t}=0$, we have sticking case, i.e., $\left|C_{t}^{T} u\right|=0$. On the other hand, if $T_{t}>0$, we have slipping case, i.e., $\left|C_{t}^{T} u\right|>0$.

This classical formulation is equivalent to the dual formulation of minimizing the total potential energy but in the incremental form. Thus the variational inequality formulation of that problem considering the surface traction $t$ and neglecting the body force $f$ is written as follows:

Minimize $F(u)=\int_{\Omega} \sigma^{T} \cdot \varepsilon d \Omega-\int_{\Gamma_{t}} u^{T} \cdot t d \Gamma$

subject to:

- The unilateral contact constraint

$C_{n}^{T} \Delta u \leq b \quad$ or $T_{n}=C_{n}^{T} \Delta u-b \leq 0 \quad$ on $\Gamma_{\mathrm{C}}$

- The stick-slip constraints

$T_{t}=\xi\left(T_{n}\right)\left|C_{t}^{T} \Delta u\right| \geq 0 \quad$ on $\Gamma_{\mathrm{C}}$

The equivalent Lagrangian formulation of the above model which constitutes both the objective functional $F(u)$ and the constraints set of Eqs. (32) and (33) is formed as

$$
\begin{aligned}
\text { Minimize } L\left(u, \lambda_{n}, \lambda_{t}\right) & =F(u)-\lambda_{n}^{T}\left(C_{n}^{T} u-b\right) \\
& -\lambda_{t}^{T} \xi\left(T_{n}\right)\left|C_{t}^{T} u\right|
\end{aligned}
$$

where $\lambda_{n}$ and $\lambda_{t}$ are the vectors of Lagrange multipliers associated with normal and tangential constraints, respectively. $\lambda_{n}$ and $\lambda_{t}$ represent the normal and tangential contact forces (reactions), respectively.

Assume that at certain level of load, the current increment is $\mathbf{J}$ and the previous increment is (J-1). Equation (34), using piecewise linearization, can be rewritten as 


$$
\begin{aligned}
\text { Minimize } \Pi\left(\Delta u, \Delta \lambda_{t}, \Delta \lambda_{n}\right) & =\int_{V} \frac{1}{2}\left(\sigma_{\mathrm{J}-1}^{T}+\Delta \sigma_{\mathrm{J}}^{T}\right)\left(\varepsilon_{\mathrm{J}-1}+\Delta \varepsilon_{\mathrm{J}}\right) d V-\left(\lambda_{n_{\mathrm{J}-1}^{T}}^{T}+\Delta \lambda_{n_{\mathrm{J}}}^{T} \mid\left[C_{n}^{T}\left(u_{\mathrm{J}-1}+\Delta u_{\mathrm{J}}\right)-b\right]\right. \\
& -\left(\lambda_{t_{\mathrm{J}-1}^{T}}^{T}+\Delta \lambda_{t_{\mathrm{J}}}^{T}\right) \xi\left(T_{n}\right)\left|C_{t}^{T}\left(u_{\mathrm{J}-1}+\Delta u_{\mathrm{J}}\right)\right|-\left(u_{\mathrm{J}-1}^{T}+\Delta u_{\mathrm{J}}^{T}\right)\left(P_{\mathrm{J}-1}+\Delta P_{\mathrm{J}}\right)
\end{aligned}
$$

Assuming that the two bodies are descritized into finite elements. To equilibrate the system, the variation of the functional given by Eq. (35) with respect to the unknown vectors, $\Delta u, \Delta \lambda_{t}$, and $\Delta \lambda_{n}$, must be vanished that results in the following equations:

$$
\begin{gathered}
K \Delta u_{\mathrm{J}}-C_{t} \Delta \lambda_{t}-C_{n} \Delta \lambda_{n_{\mathrm{J}}}=\mathrm{P}_{t o t}- \\
\int_{V} B^{T} \sigma_{\mathrm{J}-1} d V+C_{n} \lambda_{n_{\mathrm{J}-1}}+C_{t} \lambda_{t_{\mathrm{J}-1}} \\
-C_{t}^{T} u_{\mathrm{J}-1}-C_{t}^{T} \Delta u_{\mathrm{J}}=0 \\
-C_{n}^{T} \Delta u_{\mathrm{J}}+\left(b-C_{n}^{T} u_{\mathrm{J}-1}\right)=0
\end{gathered}
$$

In Eq. (36), $K$ represents the global stiffness matrix of rank $\boldsymbol{n}, K=\int_{V} B^{T} D B d V$, and $P_{\text {tot }}=P_{\mathrm{J}-1}+\Delta P_{\mathrm{J}}$ is the total applied load. Matrix $D$ for plastic elements, defined in Eq. (11) by $D_{i j k m}^{e p}$, is computed based on the stresses at the beginning of each increment. The matrix $B$ is the strain-displacement matrix that relates strain and displacement increment vectors through $\Delta \varepsilon=B \Delta u$.

The sticking pairs have $C_{t}^{T} u_{\mathrm{J}-1}=0$. Therefore, Eq. (37) turns to the following relation, which represents the compatibility conditions for displacement;

$$
-C_{t}^{T} \Delta u_{\mathrm{J}}=0
$$

The tangential constraints set, $C_{t}$, may be decomposed into two subsets, which is given by $C_{t}=C_{t a} \cup C_{t s}$

where $C_{t a}$ and $C_{t s}$ are the stick subset and slip subset, respectively. For the slipping pairs, the subset $C_{t s}$ are omitted from the compatibility conditions of Eq. (39) and the force equilibrium conditions that are given by

$$
\Delta \lambda_{t_{\mathrm{J}}}=-\mu\left|\Delta \lambda_{n_{\mathrm{J}}}\right| U_{t} /\left|U_{t}\right|
$$

are forced into the subset $C_{t s}$ of Eq. (36), where $U_{t}$ is the total relative tangential displacement. In Eq. (38), the term $\left(b-C_{n}^{T} u_{\mathrm{J}-1}\right)$ represents the updated (current) gap. Let

$$
\mathrm{P}_{\sigma}=\int_{V} B^{T} \sigma_{\mathrm{J}-1} d V
$$

Combining Eqs. (36), (38), (39), and (42), the system of equilibrium equations can be written as

$$
\left[\begin{array}{ccc}
K & -C_{t} & -C_{n} \\
-C_{t}^{T} & 0 & 0 \\
-C_{n}^{T} & 0 & 0
\end{array}\right]\left\{\begin{array}{c}
\Delta u \\
\Delta \lambda_{t} \\
\Delta \lambda_{n}
\end{array}\right\}=\left\{\begin{array}{c}
\mathrm{P}_{t o t} \\
0 \\
-\left(\mathrm{b}-C_{n}^{T} u_{\mathrm{J}-1}\right)
\end{array}\right\}-\left\{\begin{array}{c}
\mathrm{P}_{\sigma} \\
0 \\
0
\end{array}\right\}+\left[\begin{array}{ccc}
C_{t} & C_{n} & 0 \\
0 & 0 & 0 \\
0 & 0 & 0
\end{array}\right]\left\{\begin{array}{c}
\lambda_{t} \mathrm{~J}-1 \\
\lambda_{n} \\
0
\end{array}\right\}
$$

The normal contact constraints can be decomposed into active constraints, $C_{n A}$, associated with pairs in contact and inactive constraints, $C_{n N}$, associated with separated pairs.
Therefore, the normal Lagrange multipliers are decomposed into active set, $\lambda_{n A}$, and inactive set, $\lambda_{n N}$, which are equal zero. Thus, Eq. (43) will be rewritten as

$$
\left[\begin{array}{cccc}
K & -C_{t} & -C_{n A} & -C_{n N} \\
-C_{t}^{T} & 0 & 0 & 0 \\
-C_{n A}^{T} & 0 & 0 & 0 \\
-C_{n N}^{T} & 0 & 0 & 0
\end{array}\right]\left\{\begin{array}{c}
\Delta u \\
\Delta \lambda_{t} \\
\Delta \lambda_{n A} \\
\Delta \lambda_{n N}
\end{array}\right\}=\left\{\begin{array}{c}
\mathrm{P}_{t o t} \\
0 \\
0 \\
-\left(\mathrm{b}-C_{n}^{T} u_{\mathrm{J}-1}\right)
\end{array}\right\}-\left\{\begin{array}{c}
\mathrm{P}_{\sigma} \\
0 \\
0 \\
0
\end{array}\right\}+\left[\begin{array}{cccc}
C_{t} & C_{n A} & 0 & 0 \\
0 & 0 & 0 & 0 \\
0 & 0 & 0 & 0 \\
0 & 0 & 0 & 0
\end{array}\right]\left\{\begin{array}{c}
\lambda_{t_{\mathrm{J}-1}} \\
\lambda_{n A_{\mathrm{J}-1}} \\
0 \\
0
\end{array}\right\}
$$


In all the above equations, the columns of the matrices $C_{n}$ and $C_{t}$ are of the rank $\boldsymbol{n}, \boldsymbol{n}$ equals the number of displacement degrees of freedom, and for each contact condition are given by

$$
\begin{aligned}
C_{n i} & =\left[\begin{array}{llllllll}
0 & 0 & -1 & 0 \ldots \ldots . .0 & 0 & 1 & 0
\end{array}\right]^{T} \\
C_{t i} & =\left[\begin{array}{llllllll}
0 & 1 & 0 \ldots \ldots \ldots & 0 & -1 & 0 & 0
\end{array}\right]^{T}
\end{aligned}
$$

\section{SOLUTION PROCEDURE}

Once the two bodies are discretized into finite elements, the solution procedure starts according to the following steps:

If there are receding contact, i.e., pairs have initial zero gaps, the initial contact status for that pairs are assumed to be stick. With the application of full or test normal load and using the criterion of both separation and slipping described later, the contact status is adjusted incrementally with the change of one contact condition to the next trail increment. Once the receding contact status has been established, the incremental procdure will conduct as follows:

1. Initiate the increment number, i.e., $\mathrm{J}=1$, with the application of the total normal load. If there are active constraints, the total Lagrange multipliers are zero. The system represented by Eq. (44) turns into

$T \Delta=P_{t o t}$

where $T$ is the global system matrix and $\Delta$ is the unknowns vector that may be $\left\{\begin{array}{lll}\Delta u & \Delta \lambda_{t} & \Delta \lambda_{n A}\end{array}\right\}^{T}$ if there are active contact pairs or $\{\Delta u\}$ otherwise.

2. Solving this system of equations for the unknown vector $\Delta$.

3. At this stage, five potential events must be specified to determine the load required to activate it. The different scale factors required to determine each event are given as follows:

- For $C_{n N}$ calculate the normal contact constraints violation vector

$V_{\mathrm{J}, k}=T_{n}=C_{n N, k}^{T} \Delta u-g_{k}$

where, $g_{k}$ is the updated gap for the constraint number $k$. Among the set of positive values of $V \mathrm{~J}, k$, select the maximum one to may be active in the next increment that is designated by $\boldsymbol{a c}$. The adaptive contact scale factor is given by
$S_{c}=g_{a c} / C_{n N, a c}^{T} \Delta u$

- For $C_{n A}$, the criterion of separation is given by

$$
\begin{aligned}
& \lambda_{n A}=\lambda_{n A}^{* J-1}+\Delta \lambda_{n \mathrm{~A}}<0 \\
& \left|\Delta \lambda_{n A}\right|>\left|\lambda_{n A}^{* J-1}\right|
\end{aligned}
$$

where $\lambda_{n A}$ and $\lambda_{n A}^{* J-1}$ are the total normal Lagrange multipliers at the end of the current and the preceding increment, respectively. The separation scale factor is given by

$S_{r}=-\lambda_{n A}^{* J-1} / \Delta \lambda_{n A}$

Select that one has the $\operatorname{Min}\left[S_{r}\right]$ to may change from $C_{n A}$ to $C_{n N}$ at the next increment.

- For the pairs $\in C_{t a}$ at the beginning of the load increment, $\mathrm{J}$, the criterion of slipping is given by

$\left|\lambda_{t}^{* J-1}+\Delta \lambda_{t}\right| \geq \mu\left(\lambda_{n A}^{* J-1}+\Delta \lambda_{n A}\right)$

The slipping scale factor is calculated according to the following equation

$S_{f}=\left(\mu \lambda_{n A}^{* J-1}-\left|\lambda_{t}^{* J-1}\right|\right) /\left(\left|\Delta \lambda_{t}\right|-\mu \Delta \lambda_{n A}\right)$

and select the Min $\left[S_{f}\right]$ to may slip at the next increments.

- Figure (3) shows the point on the slip line which may has forward slip, I, backward slip toward the origin, II, or moves toward the stick region, III. The pairs have situation I continue to slip. Pairs have situation II tend to release or separate. Pairs have situation III tend to stick. The measure of adhesion or sticking is the dissipative energy. The dissipative energy that given by $\lambda_{t}^{* J-1} \Delta U_{t} \leq 0$ indicates continuous slipping.

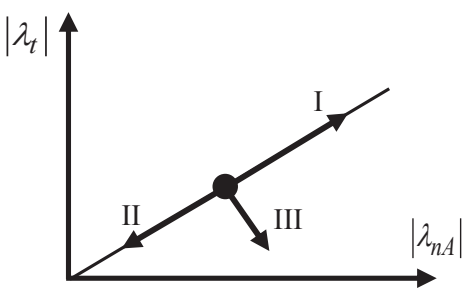

Fig. (3) Slipping pair status. 
For pairs $\in C_{t s}$ that obey the following sticking criterion

$$
\lambda_{t}^{* \mathrm{~J}-1} \Delta U_{t}>0
$$

may change to the subset $C_{t a}$ at null or zero scale factor. The stick scale factor is given by

$S_{A}=0$

- For the elements in the elastic zone, the plasticity criterion is given by

$\sigma_{e} \geq S_{y}$

For the elastic elements obey Eq. (56), the plasticity scale factors, $S_{p}$, are computed according to Eq. (16). Select the element of Min $\left[S_{p}\right]$ to may change from elastic to be plastic at the next increment.

4. The adaptive scale factor for the most probable event is chosen as follows:

$S=\operatorname{Min}\left(S_{c}, S_{p}, S_{f}, S_{r}, S_{A}\right)$

5. Update the contact status as follows :

The remaining gaps vector at the next solution step are given by

$g_{\mathrm{J}+1}=g_{\mathrm{J}}-S C_{n \mathrm{~N}}^{T} \Delta u$

The accumulated displacement field and stresses are given by

$U_{\mathrm{J}}^{*}=U_{\mathrm{J}-1}^{*}+\left.S \Delta u \quad \& \quad \sigma_{k l}^{*}\right|_{\mathrm{J}}=\left.\sigma_{k l}^{*}\right|_{\mathrm{J}-1}+\left.S \Delta \sigma_{k l}\right|_{\mathrm{J}}$

where $\left.\Delta \sigma_{k l}\right|_{\mathrm{J}}$ is computed according to Eq. (18)

for elastic elements or elastoplastic elements that use the stresses at the beginning of the increment.

The Lagrange multipliers for normal and tangential contact constraints must be updated according to

$\lambda_{n A}^{* \mathrm{~J}}=\lambda_{n A}^{* \mathrm{~J}-1}+S \Delta \lambda_{n A}$

If the contact pairs $\in C_{t a}$, then

$\lambda_{t}^{* \mathrm{~J}}=\lambda_{t}^{* \mathrm{~J}-1}+S \Delta \lambda_{t}$

If the contact pairs $\in C_{t s}$, then $\lambda_{t}^{* \mathrm{~J}}=-\mu \lambda_{n A}^{* \mathrm{~J}}\left(U_{t}^{* \mathrm{~J} J} /\left|U_{t}^{* \mathrm{~J}}\right|\right)$

where, $U_{t}^{* J}$ is the updated total relative tangential displacement.

6. Update the system of equations using Eq. (44) to accommodate the detected event.

7. Update the increment number, $\mathrm{J}=\mathrm{J}+1$.

8. Repeat steps $\mathbf{2}$ to $\mathbf{8}$ until there are no change in the events, i.e., $S=1.0$. The number of candidate contact pairs, $N_{C-n}$, must be sufficient to give the correct solution.

\section{NUMERICAL RESULTS}

\subsection{An infinite cylinder resting on a rigid surface}

The contact of an infinite cylinder resting on a rigid semi-infinite medium is shown in Figure (4). The cylinder is made of Aluminum alloy, (Al-5\%Mg), Boyer [42]. Plane strain condition is assumed. The right quarter of the cylinder is discretized into 1022 constant strain triangular elements (CST) with 571 nodes. The problem will be solved for elastic and elastoplastic analysis at different friction coefficients of $\mu=0$, 0.1 and 0.35 .

Figure (5) presents the equivalent stress distribution inside the cylinder to determine the plastic zone size. It is evident that, the increase in friction coefficient increases the plastic zone due to the increase of the tangential stress.

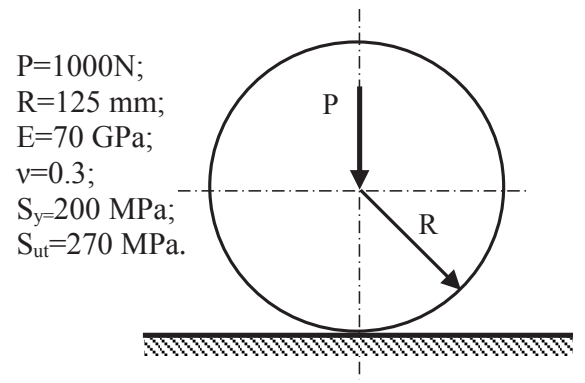

Fig. (4) A cylinder resting on rigid surface.

Johnson [43] states that for frictionless elastoplastic solution, the threshold of plastic flow will occurs at maximum contact pressure of 1.79Sy under the surface at a depth of $0.7 \mathrm{a}$, where $\mathrm{a}$ is the half contact length. The numerical and analytical results are shown in table (1) for full load elastic solution and elastoplastic solution corresponding to the onset of plasticity. The finite 


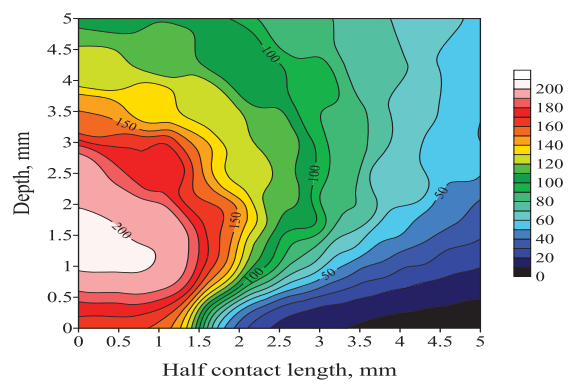

(a) $\mu=0$

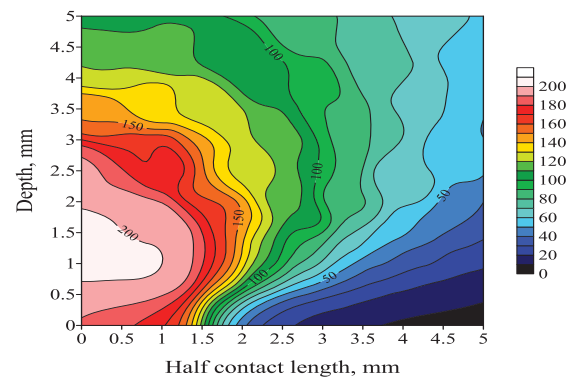

(b) $\mu=0.1$

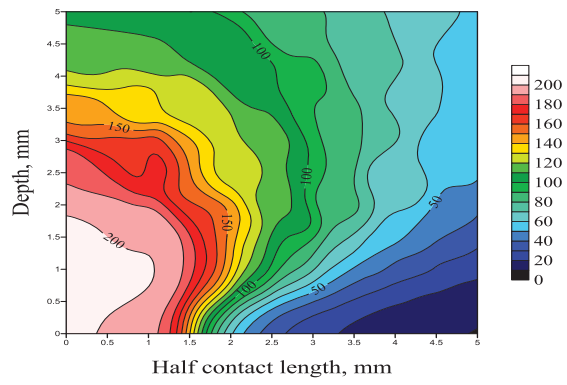

(c) $\mu=0.35$

Fig. (5) The von Mises equivalent stress distribution.

element results are in good agreement with the analytical results.

Figure (6) shows the contact pressure distribution for the elastic and the elastoplastic solutions. It is clear that the elastic solution shows small increase in the central contact pressure as the friction coefficient increase. The elastoplastic solution shows that contact pressure distribution for frictionless is nearly flat due to the decrease in the stiffness results from the plastic flow, while the contact length increases. For $\mu=0.1$, the central contact pressure increases slightly and the contact length decreases. For $\mu=0.35$, the central contact pressure drops to nearly the value of frictionless due to the expansion of the plastic zone that reaches the surface as shown in Figure (5-c).

Figure (7) presents the relative tangential displacement at the contact interface. It is shown that the RTD for elastoplastic solution less than the elastic one and the stick region increases with the increase of the friction coefficient.

Figure (8) shows the tangential stress distribution at the contact interface. It is evident that the elastic tangential stresses are greater than the elastoplastic tangential stresses due to the decrease in the stiffness that results from the plastic flow near the surface. For the elastoplastic solution, as the coefficient of friction increase the tangential stresses increase and the stick region increase.

Figure (9) shows the growth of the half contact length with the applied load fraction. The elastic solution shows as the coefficient of friction increases, the half contact length decreases and the fraction of applied load increases for the same contact length. The elastoplastic solution shows that the half contact length decreases with small increase in the friction coefficient but the half contact length increase to near the frictionless value with higher friction coefficient due to the enlargement of the plastic zone. For the same half contact length, the applied load fraction increases with small increase in the friction coefficient where the plastic zone effect is small, but it decreases with higher friction coefficient where the plastic zone effect is noticeable.

Figure (10) shows the load percent at the onset of yielding versus the friction coefficient. As $\mu$ increases the applied load at the onset of yielding decreases due to the increase in the stress that results from the friction.

\subsection{A punch intended on Half-space}

Figure (11) shows the indentation of a half space (II) by a block (I). The block is made up of elastic-perfect plastic material while the half space is made up of elastic-linear strain hardening material. The right half is discretized into 2000 CST elements with 1092 nodes. Assuming plane strain condition and friction coefficient of 0.2 .

This problem was solved elastically by Qin and Wang [14] assuming $q=1.2 \mathrm{MPa}$, and they compared their results with ABAQUS. We solve this elastic problem to validate the model for the receding contact type called the stationary contact. The results for contact stresses and the RTD are shown in Figures (12) and (13), respectively. It is cleared that the present FE model has a good agreement with the results obtained from Qin and Wang [14]. 
Table (1): Results for Elastic Full Load and Elastoplastic Frictionless at Threshold of Plasticity.

\begin{tabular}{|c|c|c|c|c|}
\hline Solution & Parameter & $\begin{array}{c}\text { Analytical } \\
\text { Johnson [43] }\end{array}$ & $\begin{array}{c}\text { FEM } \\
\text { Present results }\end{array}$ & Error \% \\
\hline \multirow{2}{*}{$\begin{array}{c}\text { Elastic } \\
(\text { El) }\end{array}$} & $\boldsymbol{a}, \mathrm{mm}$ & 1.438 & 1.43 & 0.58 \\
\cline { 2 - 5 } & Pressure, $\mathrm{MPa}$ & 442.6 & 451 & 1.86 \\
\hline $\begin{array}{c}\text { Elastoplastic } \\
(\text { El-Pl) }\end{array}$ & $\boldsymbol{a}, \mathrm{mm}$ & 1.036 & 1.08 & 4.04 \\
\cline { 2 - 5 } & Pressure, $\mathrm{MPa}$ & 358 & 323.9 & 9.5 \\
\hline
\end{tabular}

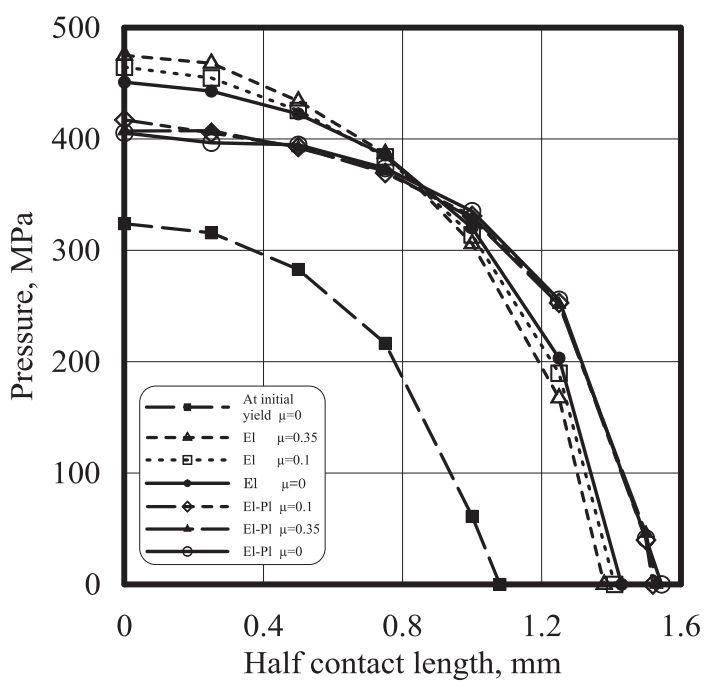

Fig. (6) Contact pressure distribution at contact interface.

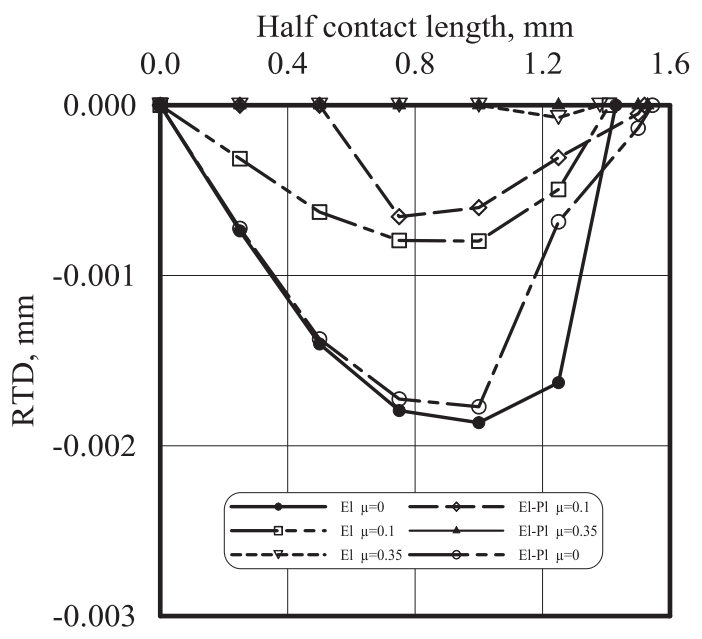

Fig. (7) Relative tangential displacement at contact interface.

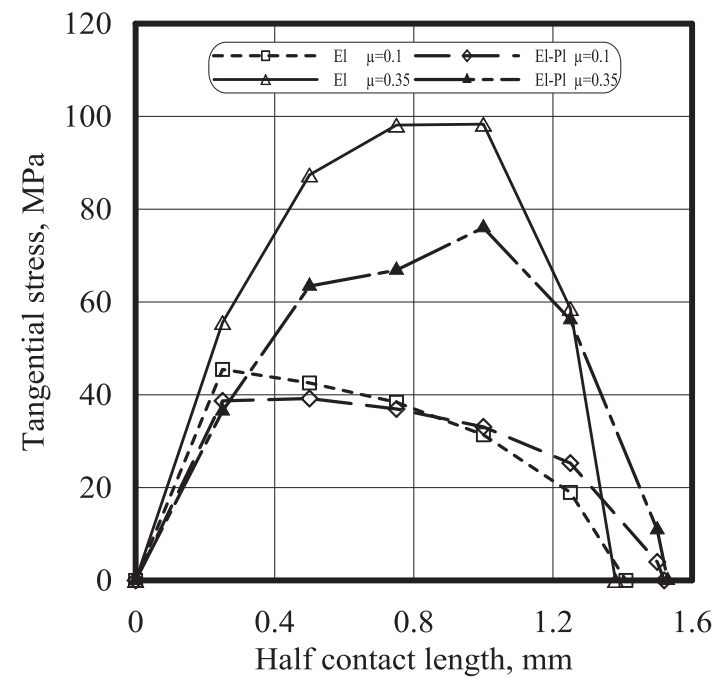

Fig. (8) Tangential stress distribution at contact interface.

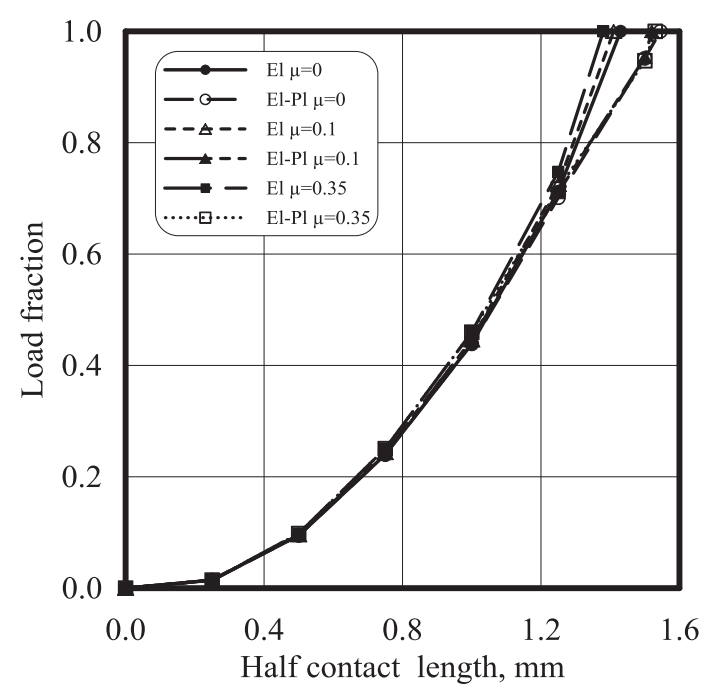

Fig. (9) Growth of half contact length with load fraction. 


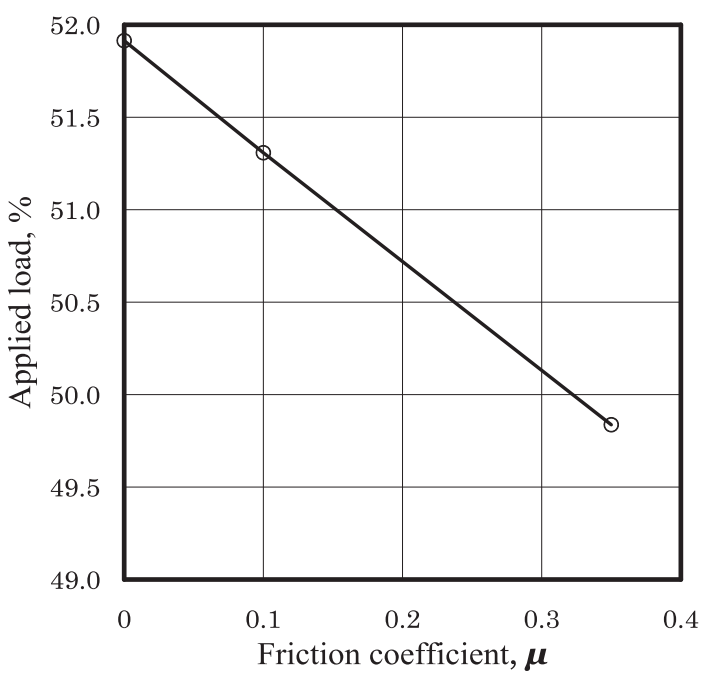

Fig. (10) Load percent at onset of yield vs. the friction coefficient.

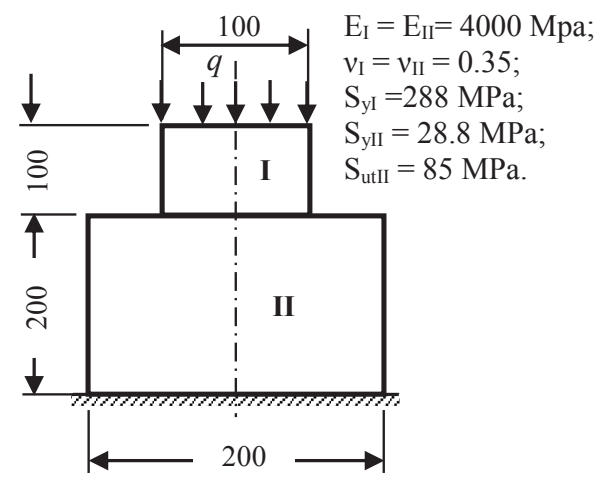

Fig. (11) Two deformable blocks under distributed load.

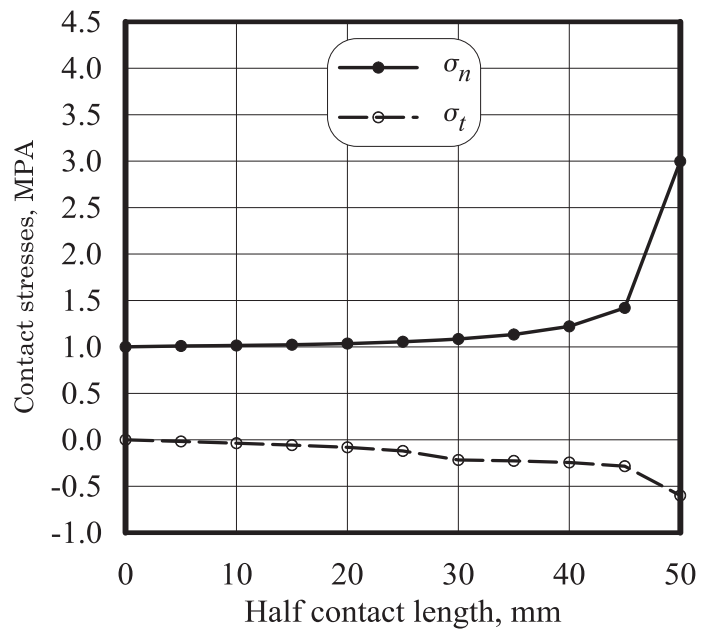

(a) Present FE Model
Re-assuming plane stress condition, the elastoplastic solution for this problem with $\mu=0$ and 0.2 are carried out for $q=36 \mathrm{MPa}$ to produce considerable plastic effects.

Figure (14) shows the contact pressure distribution versus the half contact length. It is nearly flat but rises rapidly near the lower end point of contact, singularity point, due to the stress concentration at that zone. Central contact pressure for $\mu=0.2$ is slightly larger than that of $\mu=0$.

Figure (15) shows the variation of RTD with the increase of loading. It is evident that as the load increases, the slip zone decreases. This phenomenon is consistence with the results published by Zhu [27].

Figure (16) presents the variation of the tangential stress with the increase of the load. As the load increases, both the tangential stress and the stick zone increase. It is evident from Figures (15) and (16) that the tangential stress opposes the RTD.

\section{CONCLUSIONS}

An adaptive incremental approach handling nonlinear elastoplastic frictional contact problems is developed using the FEM and piecewise linearization technique. The problem is formulated as an incremental convex programming model under inequality contact constraints. The Lagrange multiplier approach is used to enforce the contact constraints. Friction is

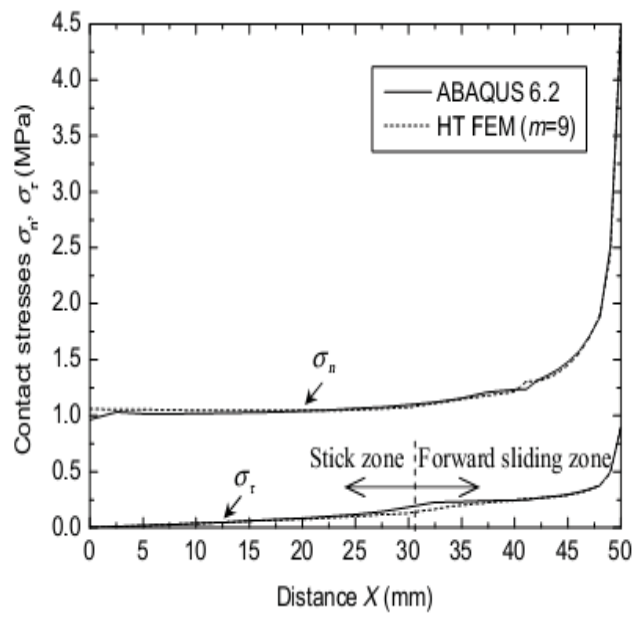

(b) Qin and Wang [14]

Fig. (12) Contact stresses along the interface. 


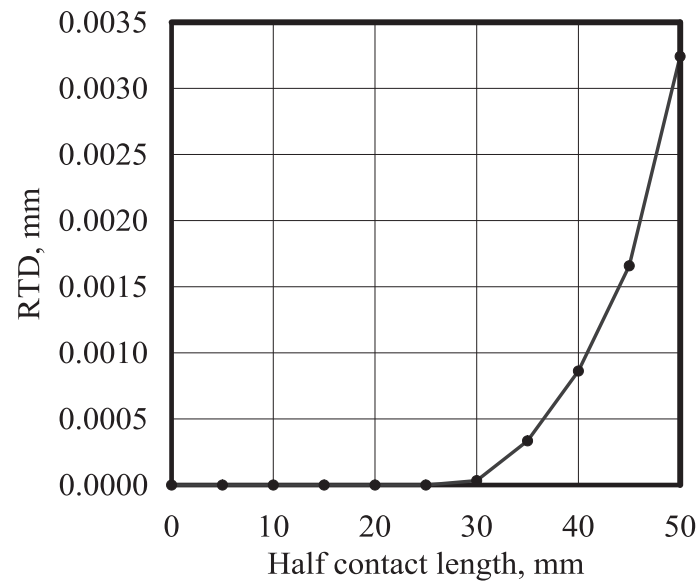

(a) Present FE Model

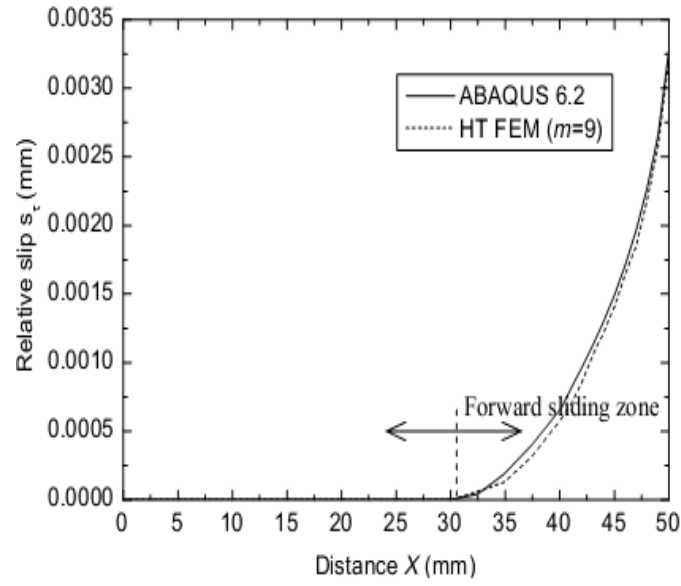

(b) Qin and Wang [14]

Fig. (13) Relative tangential displacement at the contact interface.

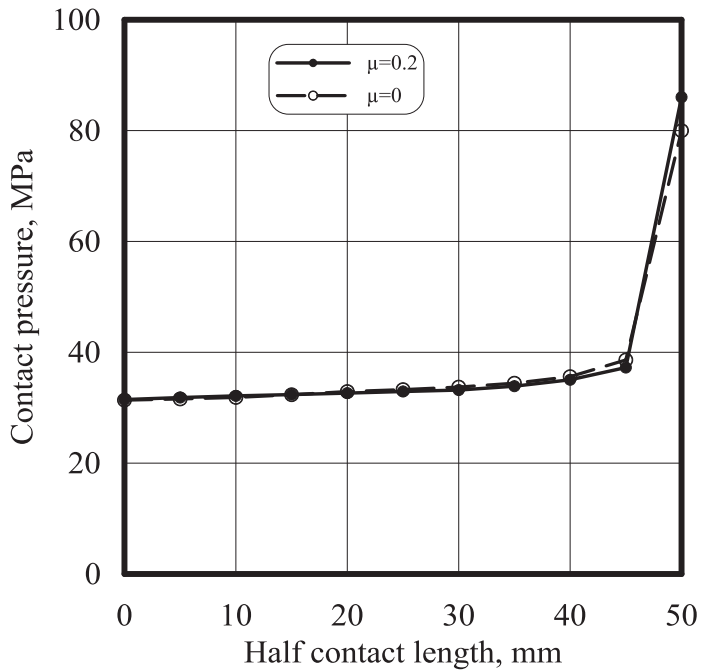

Fig. (14) Contact pressure distribution.

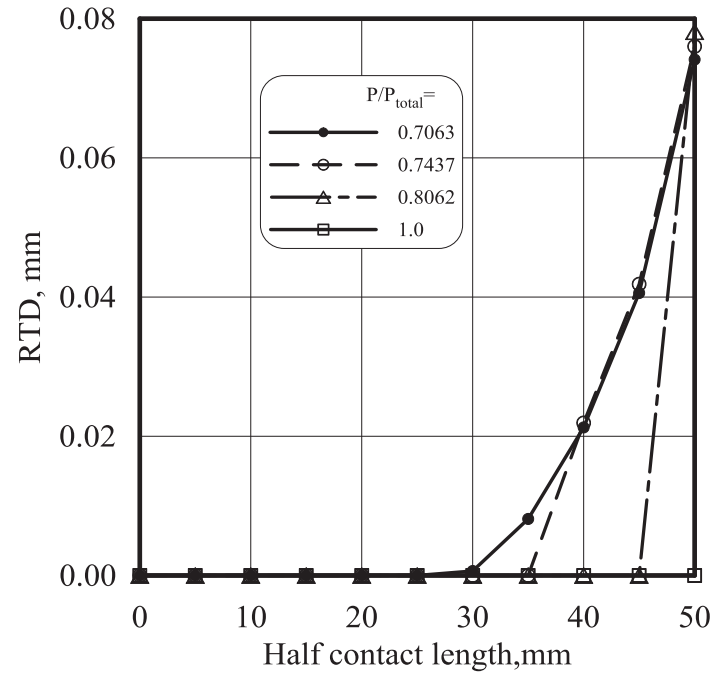

Fig. (15) RTD distribution along the interface.

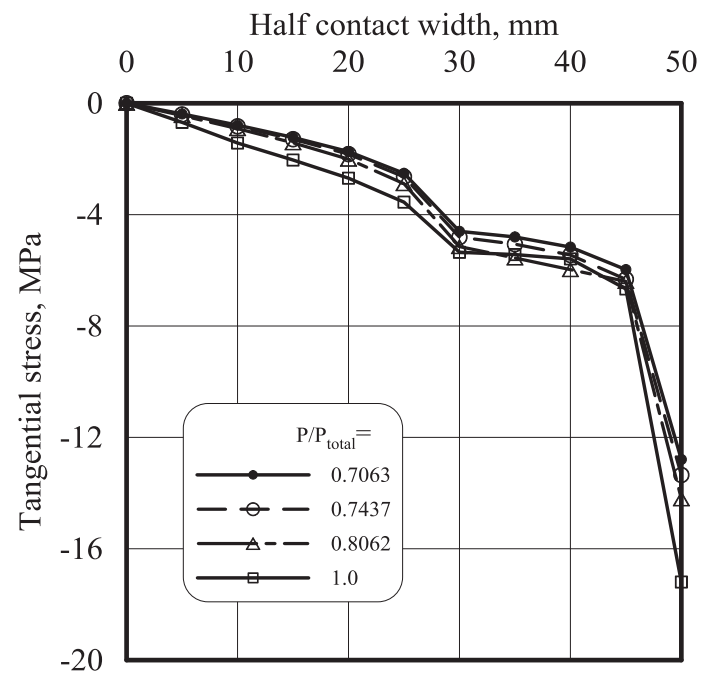

Fig. (16) Tangential stress distribution along the interface. 
simulated using the classical Coulomb's friction law. The incremental stress-strain constitutive relations based on the Prandtl-Reuss flow rule and von Mises yield criterion are adopted.

The developed adaptive incremental procedure is applied successfully to the elastic and elastopalstic contact problems of a cylinder resting on a rigid frictional surface, and the indentation problem of a half-space by an elastic block under normal load. The obtained results agree well with the results available in the literature. The elastic results show that as the friction coefficient increases the central contact pressure undergoes a small increase with small decrease of the contact area. Elastoplastic analysis shows that increasing the friction coefficient results in a slight increase in the central contact pressure until the friction coefficient reaches a higher values, the pressure distribution returns nearly to the frictionless one. As the friction coefficient increases, the plastic zone enlarges and the slip region decreases. Additionally, the adaptive incremental procedure has the ability to determine the load capacity required for certain contact length and size of plastic zone that is important in the design analysis.

\section{REFERENCES}

1. N. Okamoto and M. Nakazawa, "Finite element incremental contact analysis with various frictional conditions", International Journal for Numerical Methods in Engineering, 14 (1979) 337-357.

2. J. T. Oden and E. Pires, "Nonlocal and nonlinear friction laws and variational principles for contact problems in elasticity", Journal of Applied Mechanics, 50 (1983) 67-76.

3. K. J. Bathe and A. Chaudhary, "A solution method for planar and axisymmetric contact problems", International Journal for Numerical Methods in Engineering, 21 (1985) 65-88.

4. W. Zhong and S. Sun, "A parametric quadratic programming approach to elastic contact problems with friction", Computers \& structures, 32 (1989) 37-43.

5. F. Paris and J. Garrido, "An incremental procedure for friction contact problems with the boundary element method", Engineering analysis with boundary elements, 6 (1989) 202-213.

6. J. Garrido, A. Foces and F. Paris, "An incremental procedure for three-dimensional contact problems with friction", Computers \& structures, 50 (1994) 201-215.
7. S. S. Lee, "A computational method for frictional contact problem using finite element method", International Journal for numerical methods in engineering, 37 (1994) 217-228.

8. A. Saleeb, K. Chen and T. Chang, "An effective two-dimensional frictional contact model for arbitrary curved geometry", International journal for numerical methods in engineering, 37 (1994) 1297-1321.

9. F. F. Mahmoud, S. S. Ali-Eldin, M. M. Hassan and S. A. Emam, "An incremental mathematical programming model for solving multi-phase frictional contact problems", Computers \& structures, 68 (1998) 567-581.

10. A. R. Mijar and J. S. Aroar, "A new frictional contact problem formulation using Augmented Lagrangian Method", the $42^{\text {nd }}$ AIAA Structures, Structural Dynamics, and Materials Conference, Seattle, WA, (2001) 504-514.

11. C. Renaud and Z.-Q. Feng, "BEM and FEM analysis of Signorini contact problems with friction", Computational mechanics, 31 (2003) 390-399.

12. M. M. Hassan and F. F. Mahmoud, "A generalized adaptive incremental approach for solving inequality problems of convex nature", Structure Engineering and Mechanics, 184(2004) 461-474.

13.S. A. Mohamed, M. M. Helal, F. F. Mahmoud, "An incremental convex programming model of the elastic frictional contact problems", Structural Engineering and Mechanics, 23-4 (2006).

14. Q.-H. Qin and K.-Y. Wang, "Application of hybrid-Trefftz finite element method to frictional contact problems", Computer Assisted Mechanics and Engineering Sciences, 15 (2008) 319-336.

15. Y. J. Ahn and J. Barber, "Response of frictional receding contact problems to cyclic loading", International Journal of Mechanical Sciences, 50 (2008) 1519-1525.

16. J. Dundurs, "Properties of elastic bodies in contact, The mechanics of the contact between deformable bodies", (1975) 54-66.

17. B. Kanber and N. Demirhan, "Finite element analysis of frictional contact of elastic solids with thin amd moderately thick coatings", Turkish J Eng Env Sci (2013) 37: 162-177.

18. W. S. Abdalla, S. S. Ali-Eldin and M. R. Ghazy, "Modeling and solution of frictional contact problems using finite element method", Emirates Journal for Engineering Research, 18 (1) (2013) 25-32. 
19. Y. Yamada, N. Yoshimura and T. Sakurai, "Plastic stress-strain matrix and its application for the solution of elastic-plastic problems by the finite element method", International Journal of Mechanical Sciences, 10 (1968) 343-354.

20. O. Zienkiewicz, S. Valliappan and I. King, "Elasto-plastic solutions of engineering problems 'initial stress', finite element approach", International Journal for Numerical Methods in Engineering, 1 (1969) 75-100.

21. G. Dumas and C. Baronet, "Elastoplastic indentation of a half-space by an infinitely long rigid circular cylinder", International Journal of Mechanical Sciences, 13 (1971) 519-530.

22. H. Nagaraj, "Elastoplastic contact of bodies with friction under normal and tangential loading", Journal of tribology, 106 (1984) 519526.

23. J. Cheng and N. Kikuchi, "An analysis of metal forming processes using large deformation elastic-plastic formulations", Computer Methods in Applied Mechanics and Engineering, 491985 71-108.

24. K. Komvopoulos, "Elastic-plastic finite element analysis of indented layered media", Journal of Tribology, 111 (1989) 430-439.

25. H. Wang, X. Yang, H. Bangert, P. Torzicky and L. Wen, "Two-dimensional finite element method simulation of Vickers indentation of hardness measurements on TiN-coated steel", Thin Solid Films, 214 (1992) 68-73.

26. P. Montmitonnet, M. Edlinger and E. Felder, "Finite element analysis of elastoplastic indentation. I: Homogeneous media", Journal of Tribology, 115 (1993) 10-14.

27. C. Zhu, "A finite element-mathematical programming method for elastoplastic contact problems with friction", Finite elements in analysis and design, 20 (1995) 273-282.

28. D. Pantuso, K. -J. Bathe and P. A. Bouzinov, "A finite element procedure for the analysis of thermo-mechanical solids in contact", Computers \& Structures, 75 (2000) 551-573.

29. L. Menezes and C. Teodosiu, "Threedimensional numerical simulation of the deepdrawing process using solid finite elements", Journal of materials processing technology, 97 (2000) 100-106.

30. D. Sheng, P. Wriggers and S. W. Sloan, "Improved numerical algorithms for frictional contact in pile penetration analysis", Computers and Geotechnics, 33 (2006) 341-354.
31. R. Vijaywargiya and I. Green, "A finite element study of the deformations, forces, stress formations, and energy losses in sliding cylindrical contacts", International Journal of Non-Linear Mechanics 42 (2007) 914-927.

32. M. Anahid and A. Khoei, "New development in extended finite element modeling of large elasto-plastic deformations", International Journal for Numerical Methods in Engineering, 75 (2008) 1133-1171.

33. A. A. Bandeira, P. M. Pimenta, B. V. Jr. Alberto and Sá R. Jr. Armando, "Numerical simulation of contact problems under large $3 \mathrm{~d}$ elastoplastic deformation", $2^{\text {nd }}$ International Conference on Engineering Optimization, September 6 - 9, 2010, Lisbon, Portugal.

34. C. J. Adam and M. V. Swain, "The effect of friction on indenter force and pile-up in numerical simulations of bone nanoindentation", In: CMBBE2010: $9^{\text {th }}$ International Symposium on computer methods in Biomechanics and Biomedical Engineering, Valencia, Spain, February 4-27, 2010.

35. E. H. Boudaia, L. Bousshine and A. Chaaba, "Solving frictional contact problems within the bipotential framework", Key Engineering Materials, 498 (2012) 55-66.

36. B. Chatterjee and P. Sahoo, "Effect of strain hardening on elastic-plastic contact of a deformable sphere against a rigid flat under full stick contact condition", Advances in Tribology, 2012 (2012).

37. R. Weyler, J. Oliver, T. Sain and J. C. Cante, "On the contact domain method: A comparison of penalty and Lagrange multiplier implementations", Comput. Methods Appl. Mech. Engrg. 205-208 (2012) 68-82.

38. A. Kumar, T. Staedler and X. Jiang, "Effect of normal load and roughness on the nanoscale friction coefficient in the elastic and plastic contact regime", Bellstein J. Nanotechnol. 4 (2013) 66-71.

39. K. Poulios and P. Klit, "Implementation and applications of a finite-element model for the contact between rough surfaces"', Wear 303 1-2 (2013) 1-8.

40. V. L. Popov, "Contact mechanics and friction: physical principles and applications", Springer, (2010).

41. A. Mendelson, "Plasticity: theory and application", Macmillan New York, (1968).

42. H. E. Boyer, "Atlas of Stress-Strain Curves", ASM International, (2002). 
43.K. L. Johnson, "Contact Mechanics, 3ed,

Cambridge University Press, Cambridge, (1992).

\section{Appendix $[\mathbf{A}]$}

\section{Incremental Elastoplastic Flow Rule}

Let $\sigma_{x}, \sigma_{y}, \sigma_{z}, \tau_{x y}, \tau_{y z}$ and $\tau_{z x}$ are the current or total values of the stresses. Thus

$$
\begin{aligned}
& S_{x}=\sigma_{x}-0.5\left(\sigma_{y}+\sigma_{z}\right), \\
& S_{y}=\sigma_{y}-0.5\left(\sigma_{x}+\sigma_{z}\right), \text { and } \\
& S_{z}=\sigma_{z}-0.5\left(\sigma_{x}+\sigma_{y}\right)
\end{aligned}
$$

Three dimensional elastoplastic incremental strain-stress relation in explicit matrix form is given by

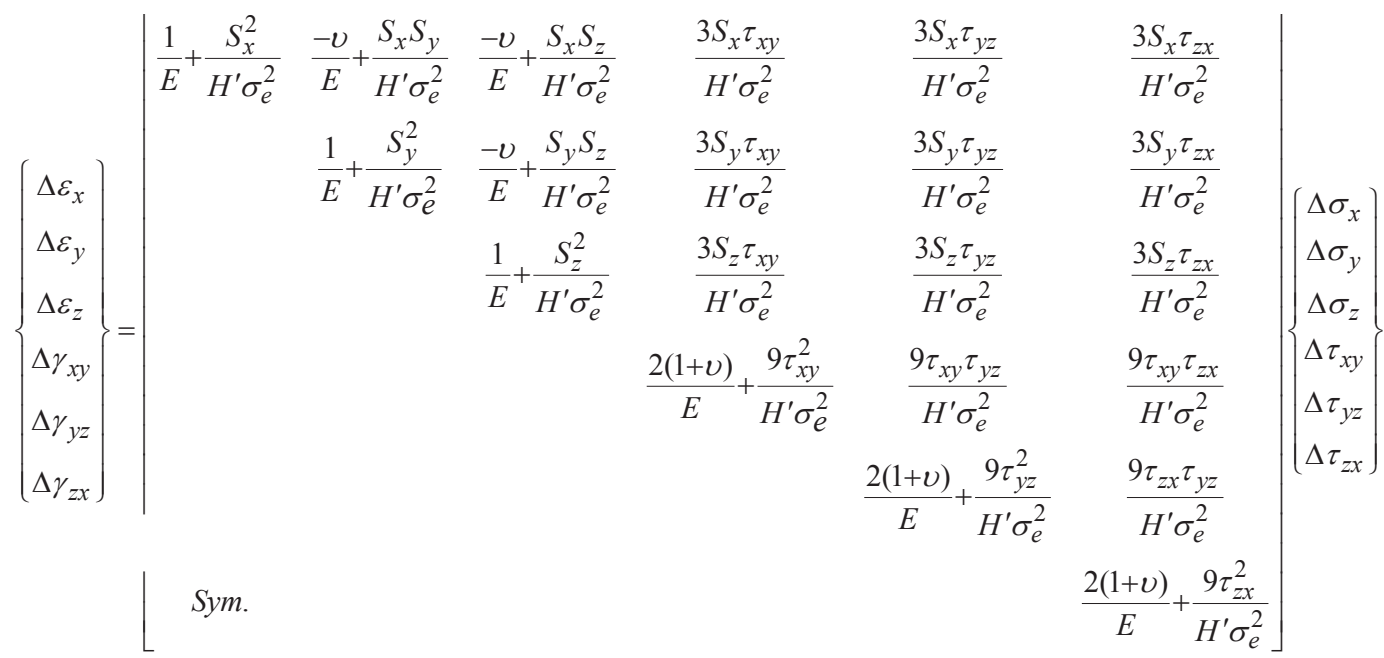

- Special Forms of The Elastoplastic Relationship

Three dimensional elastoplastic incremental strain-stress relations, represented by Eq. (A.2), can be reduced to the two-dimensional analysis as follows:

For the plane stress distribution, Eq. (A.2), is reduced to

$$
\left\{\begin{array}{c}
\Delta \varepsilon_{x} \\
\Delta \varepsilon_{y} \\
\Delta \gamma_{x y}
\end{array}\right\}=\left[\begin{array}{ccc}
\frac{1}{E}+\frac{\left(\sigma_{x}-0.5 \sigma_{y}\right)^{2}}{H^{\prime} \sigma_{e}^{2}} & \frac{-v}{E}+\frac{\left(\sigma_{x}-0.5 \sigma_{y}\right)\left(\sigma_{y}-0.5 \sigma_{x}\right)}{H^{\prime} \sigma_{e}^{2}} & \frac{3\left(\sigma_{x}-0.5 \sigma_{y}\right) \tau_{x y}}{H^{\prime} \sigma_{e}^{2}} \\
\frac{1}{E}+\frac{\left(\sigma_{y}-0.5 \sigma_{x}\right)^{2}}{H^{\prime} \sigma_{e}^{2}} & \frac{3\left(\sigma_{y}-0.5 \sigma_{x}\right) \tau_{x y}}{\prime_{e}^{2}} \\
\text { Sym. } & \frac{2(1+v)}{E}+\frac{9 \tau_{x y}^{2}}{H^{\prime} \sigma_{e}^{2}}
\end{array} \mid\left\{\begin{array}{l}
\Delta \sigma_{x} \\
\Delta \sigma_{y} \\
\Delta \tau_{x y}
\end{array}\right\}\right.
$$

For the plane Strain distribution, using Eqs. (A.1, 2), let

$$
\begin{aligned}
& \mathrm{C}_{1}=\frac{1}{E}+\frac{S_{x}^{2}}{H^{\prime} \sigma_{e}^{2}}, \quad \mathrm{C}_{2}=\frac{-v}{E}+\frac{S_{x} S_{y}}{H^{\prime} \sigma_{e}^{2}}, \quad \mathrm{C}_{3}=\frac{-v}{E}+\frac{S_{x} S_{z}}{H^{\prime} \sigma_{e}^{2}}, \mathrm{C}_{4}=\frac{3 S_{x} \tau_{x y}}{H^{\prime} \sigma_{e}^{2}}, \\
& \mathrm{C}_{5}=\frac{1}{E}+\frac{S_{y}^{2}}{H^{\prime} \sigma_{e}^{2}}, \quad \mathrm{C}_{6}=\frac{-v}{E}+\frac{S_{y} S_{z}}{H^{\prime} \sigma_{e}^{2}}, \quad \mathrm{C}_{7}=\frac{3 S_{y} \tau_{x y}}{H^{\prime} \sigma_{e}^{2}}, \quad \mathrm{C}_{8}=\frac{1}{E}+\frac{S_{z}^{2}}{H^{\prime} \sigma_{e}^{2}}, \\
& \mathrm{C}_{9}=\frac{3 S_{z} \tau_{x y}}{H^{\prime} \sigma_{e}^{2}}, \text { and } \mathrm{C}_{10}=\frac{2(1+v)}{E}+\frac{9 \tau_{x y}^{2}}{H^{\prime} \sigma_{e}^{2}}
\end{aligned}
$$


We find that

$\Delta \sigma_{z}=-\frac{C_{3}}{C_{8}} \Delta \sigma_{x}-\frac{C_{6}}{C_{8}} \Delta \sigma_{y}-\frac{C_{9}}{C_{8}} \Delta \tau_{x y}$
(A.5)

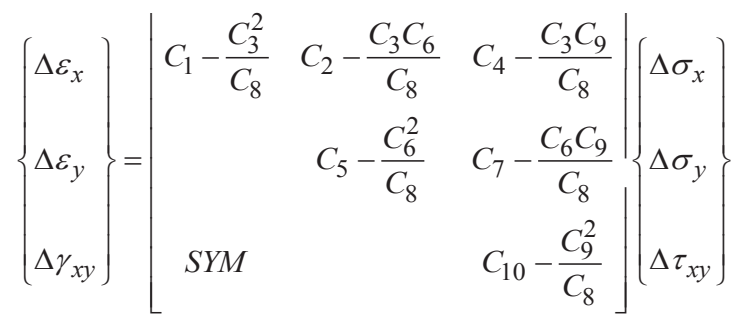

